\title{
Avaliação do perfil epidemiológico de pacientes com doença reumatológica em um centro de especialidades de Marabá, Pará, Brasil
}

\author{
Evaluation of the epidemiological profile of patients with rheumatologic disease in a specialty \\ center in Maraba, Para, Brazil \\ Evaluación del perfil epidemiológico de pacientes con enfermedad reumatológica en un centro de \\ especialidades de Marabá, Pará, Brasil
}

Recebido: 17/08/2021 | Revisado: 26/08/2021 | Aceito: 28/08/2021 | Publicado: 30/08/2021

\author{
Ana Thais dos Santos Quirino \\ ORCID: https://orcid.org/0000-0002-9091-3131 \\ Universidade do Estado do Pará, Brasil \\ E-mail: atquirino19@gmail.com \\ Victor Felipe de Almeida Leal \\ ORCID: https://orcid.org/0000-0001-8072-1729 \\ Universidade do Estado do Pará, Brasil \\ E-mail: victorf.almeida2@gmail.com \\ Cilene Aparecida de Souza Melo \\ ORCID: https://orcid.org/0000-0003-1170-6466 \\ Universidade do Estado do Pará, Brasil \\ E-mail: souza_cilene927@hotmail.com
}

\begin{abstract}
Resumo
Objetivo: Caracterizar pacientes acometidos por doença reumática em um centro de especialidades do interior amazônico. Metodologia:Estudo retrospectivo transversal, observacional, com abordagem quantitativa de uma amostra de 182 prontuários de indivíduos em acompanhamento no serviço de reumatologia no período entre 31 de dezembro de 2019 a 01 de janeiro de 2020 em um centro de especialidades localizado no município de Marabá, Pará, Brasil. Resultados: Observou-se que houve uma predominância de pacientes do sexo feminino (92,31\%), com idade entre 61 a 70 anos (37,36\%). O principal diagnóstico encontrado foi fibromialgia seguido de artrite reumatoide. As principais comorbidades encontradas foram HAS e DM e o principal medicamento utilizado foi a pregabalina, em concordância com o principal diagnóstico. Do contrário de inúmeros estudos acerca do perfil de pacientes reumatológicos, todos os pacientes apresentaram tempo de tratamento inferior a 5 anos. Conclusão:Através dos resultados, pode-se constatar que o município de Marabá acompanha as estatísticas nacionais no âmbito da epidemiologia das doenças reumatológicas. Com isso, o entendimento desse perfil pode proporcionar melhorias a médio e longo prazo no acompanhamento dos pacientes da área da reumatologia, possibilitando uma integralidade do cuidado.
\end{abstract}

Palavras-chave: Perfil de saúde; Reumatologia; Saúde global; Doença crônica.

\begin{abstract}
Objective: To characterize patients suffering from rheumatic disease in a specialty center in the interior of the Amazon. Methodology: Retrospective, observational, cross-sectional study with a quantitative approach of a sample of 182 medical records of individuals being monitored at the rheumatology service in the period between December 31, 2019 and January 1, 2020 in a specialty center located in the city of Maraba, Para, Brazil. Results: It was observed that there was a predominance of female patients $(92.31 \%)$, aged between 61 and 70 years $(37.36 \%)$. The main diagnosis found was fibromyalgia followed by rheumatoid arthritis. The main comorbidities found were SAH and DM and the main drug used was pregabalin, in agreement with the main diagnosis. Contrary to numerous studies on the profile of rheumatologic patients, all patients had a treatment time of less than 5 years. Conclusion: Through the results, it can be seen that the municipality of Marabá follows national statistics in the scope of the epidemiology of rheumatologic diseases. Thus, the understanding of this profile can provide medium and long-term improvements in the monitoring of patients in the field of rheumatology, enabling comprehensive care.
\end{abstract}

Keywords: Health profile; Rheumatology; Global health; Chronic disease.

\section{Resumen}

Objetivo: Caracterizar a los pacientes que padecen enfermedad reumática en un centro de especialidades del interior de la Amazonía. Metodología: Estudio retrospectivo, observacional, transversal con abordaje cuantitativo de una muestra de 182 historias clínicas de individuos en seguimiento en el servicio de reumatología en el período 
comprendido entre el 31 de diciembre de 2019 y el 1 de enero de 2020 en un centro de especialidades ubicado en la ciudad. de Marabá, Pará, Brasil. Resultados: Se observó un predominio de pacientes del sexo femenino $(92,31 \%)$, con edades comprendidas entre 61 y 70 años $(37,36 \%)$. El principal diagnóstico encontrado fue fibromialgia seguida de artritis reumatoide. Las principales comorbilidades encontradas fueron HSA y DM y el principal fármaco utilizado fue pregabalina, de acuerdo con el diagnóstico principal. Al contrario de numerosos estudios sobre el perfil de los pacientes reumatológicos, todos los pacientes tuvieron un tiempo de tratamiento inferior a 5 años. Conclusión: A través de los resultados se puede apreciar que el municipio de Marabá monitorea las estadísticas nacionales en el ámbito de la epidemiología de las enfermedades reumatológicas. Así, la comprensión de este perfil puede aportar mejoras a medio y largo plazo en el seguimiento de los pacientes en el ámbito de la reumatología, posibilitando una atención integral.

Palabras clave: Perfil de salud; Reumatología; Salud global; Enfermedad crónica.

\section{Introdução}

Doenças reumatológicas, de acordo com a sociedade brasileira de reumatologia (SBR), são um conjunto de patologias que afetam o aparelho locomotor como: ossos, articulações, cartilagens, músculos, tendões e ligamento. Podendo afetar outras áreas do corpo, além de atingir pessoas de qualquer idade independente de sexo ou raça. Alguns fatores podem ser considerados no agravamento dos múltiplos sintomas ocasionados por esse conjunto de doenças. Fatores genéticos, depressão, sedentarismo, obesidade e estilo de vida impactam diretamente no agravamento desses sintomas. Atualmente, de acordo com dados fornecidos pelo Ministério da Saúde, 15 milhões de pessoas sofrem com alguma doença reumatológica (Silva, Laranjeira, Silva \& Lorena, 2017).

As patologias de caráter reumatológico, na maioria dos casos, possui evolução de caráter crônico e costumam ter progressão degenerativa. A forma de evolução, prognóstico e tratamento do paciente portador de doença reumatoide depende da patologia que o acomete, além dos fatores de risco que podem ocasionar a rápida evolução da doença, alguns mecanismos fisiopatológicos de determinadas doenças reumatoides. Os sintomas clássicos deste conjunto de doenças acometem principalmente o aparelho locomotor, sintomas como dor, limitação ao movimento, rigidez nas articulações atingem diretamente na percepção de qualidade de vida do paciente. Tais sintomas podem acarretar em quadros depressivos devido ao isolamento social, além da mudança de sua percepção como ser ativo em sociedade (Silva, 2019)

A medicina avança a passos largos todos anos, os prognósticos destes pacientes mostram-se promissores. Nota-se também a maior busca por informações referentes as doenças reumáticas, quanto maior a carga de informações, menor a possibilidade de complicações e possíveis internações relacionadas a essas doenças, tendo em vista que esses pacientes conviverão cronicamente com a enfermidade. É de conhecimento da população científica que pacientes que possuem alguma doença de cunho reumatológico tem maiores chances de desenvolver doenças de caráter infectocontagiosas se equiparados a população geral. Isto ocorre devido a dois fatores: terapia imunossupressora utilizada na maioria dos tratamentos de doenças reumáticas e imunossupressão relacionada aos mecanismos fisiopatológicos das doenças reumáticas (Oliveira \& Ramos, 2019).

A busca do perfil epidemiológico de pacientes com doenças reumáticas faz com que seja possível traçar futuras medidas de tratamento e profilaxia dos principais sintomas e sinais das doenças. Informações como sexo, idade, faixa etária e aspectos socioeconômicos são de suma importância, pois a partir destes é possível avaliar se algum desses aspectos pode impactar no meio social de casa paciente (Carvalho, Costa, \& Silva, 2018).

Inúmeros estudos demonstram que as doenças reumáticas causam inúmeros impactos negativos na vida de seus portadores, pois devido seu amplo espectro clínico faz com que os acometidos pelas doenças possam ter desde pequenas limitações físicas causadas pela dor até mesmo pela a incapacidade funcional (Rodrigues, Rodrigues, Nogueira, Souza \& Sousa, 2019). Sendo assim, o estudo em questão mostra-se viável, pois é preciso saber: o sexo, a idade ou qualquer outro fator epidemiológico podem agravar sinais e sintomas das doenças reumatológicas? Além disso, dentre as doenças reumáticas, há algum grupo suscetível a ter maiores chances de desenvolver formas graves de alguma doença reumatológica? 


\section{Metodologia}

Trata-se de estudo retrospectivo observacional, transversal baseado na análise de variáveis quantitativas (Estrela, 2018). A pesquisa foi realizada no município de Marabá, região Sudeste do estado do Pará. Segundo estimativa do IBGE para o ano de 2019, a população estimada é de 283.542 habitantes e a densidade populacional corresponde a 15,45 habitantes/km² . Quanto aos aspectos de saúde pública, a cidade possui 41 estabelecimentos de saúde e uma taxa de mortalidade infantil de 18.68/1000 nascidos vivos, figurando na $40^{\circ}$ posição entre os municípios do Estado do Pará.

A amostra foi composta por 182 indivíduos em acompanhamento de doença reumatológica no local de estudo. Foram analisados os prontuários dos pacientes atendidos no ambulatório de reumatologia no período entre 01 de janeiro de 2019 a 31 de dezembro de 2020. Foram coletados dados de sexo, idade, tempo de acompanhamento, comorbidades, diagnóstico e tratamento abordado contidos no prontuário. Foram excluídos da pesquisa prontuários de pacientes com dados incompletos ou com dados ausentes.

Após a coleta dos dados, foi realizada a análise e interpretação dos mesmos e submetidos à organização em planilhas digital no programa Microsoft Excel para análise estatística descritiva, utilizando o programa para cálculo de frequências absolutas, relativas e média dos dados. Em seguida, os dados foram organizados em gráficos e tabelas para melhor entendimento.

A pesquisa foi aprovada pelo Comitê de Ética em Pesquisa da Universidade do Estado do Pará antes do prosseguimento da mesma, obedecendo aos critérios de ética em pesquisa conforme resolução $\mathrm{N}^{\mathrm{o}} 466 / 12$ do Conselho Nacional de Saúde - CNS, além de manter-se alinhado ao código de Nuremberg de 1949. (CAAE: 47621221.4.0000.8607).

\section{Resultados e Discussão}

Foram coletados dados de 182 prontuários disponíveis no sistema de prontuários eletrônicos. Observou-se uma maior prevalência desse grupo de patologias no sexo feminino (92,31\%) se comparadas a pacientes do sexo masculino (7,61\%), resultados compatíveis com a literatura, que demonstram um acometimento maior em mulheres (Carvalho, Costa, \& Silva, 2013). A predominância das doenças reumatológicas em mulheres ainda não é totalmente compreendida, no entanto, acreditase que as oscilações hormonais possuam papel importante no surgimento das doenças reumatológicas principalmente durante o período do climatério, no qual observa-se um aumento na prevalência dessas patologias nas mulheres a partir dos 40 anos em comparação aos homens de mesma idade (Martins, Carrilho, Alves, Castilho, \& Cerri, 2016).

O grupo etário com maior prevalência neste conjunto de doenças se encontra entre 61 e 70 anos (Figura 1), o que se apresenta em desacordo com a maioria dos estudos publicados anteriormente, que demonstraram uma média de idade entre 40 a 59 anos (Bonetti, Souza, \& Resmini, 2020). A preferência das doenças reumatológicas pelas idades mais avançadas podem estar relacionadas ao desgaste do tecido conjuntivo durante o processo de envelhecimento associada aos estresses mecânicos ao qual o indivíduo se expõe ao longo da vida, levando ao aparecimento dos sintomas nas idades mais avançadas (Silva, 2017). Outro aspecto importante é a influência da menopausa no surgimento mais precoce das doenças reumatológicas em mulheres a partir de 40 anos, cujo fator hormonal está diretamente interligado, uma vez que o estrógeno parece conferir fator protetor contra algumas doenças autoimunes e osteoarticulares (Rodrigues, Rodrigues, Nogueira, Souza \& Sousa, 2019). 
Figura 1 - Pacientes reumatológicos, segundo faixa etária.

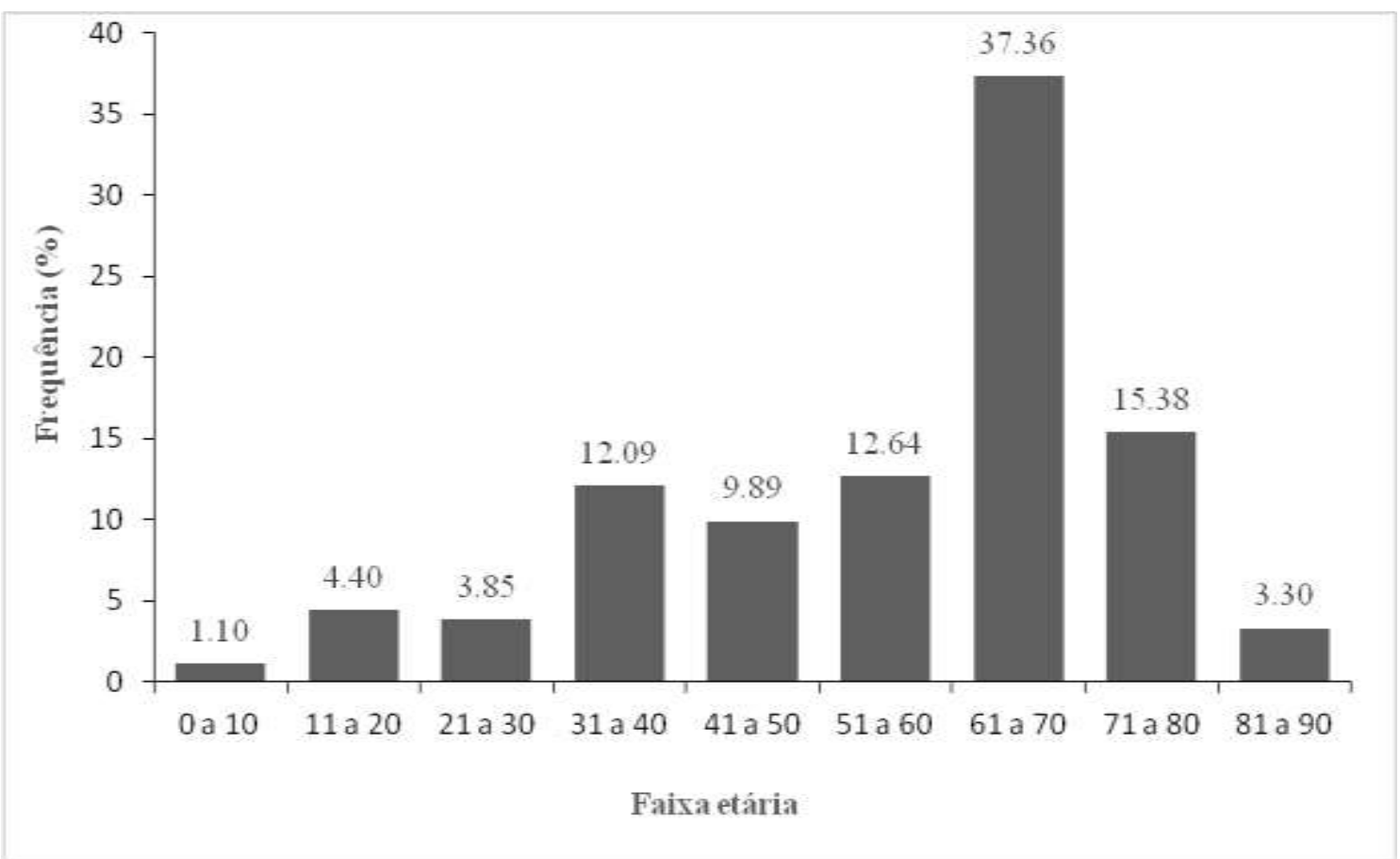

Fonte: Acervo da pesquisa (2021).

Dentre o grupo de doenças (Tabela 1), as que obtiverem maior prevalência foram fibromialgia (56 pacientes), artrite reumatoide (37 pacientes), osteoartrose (34 pacientes), lúpus eritematoso sistêmico (19 pacientes) e afecções ligadas a gravidez (8 pacientes), o que se apresentou de acordo com o estudo de Montecucco, Cavagna e Caporali (2009) o qual evidenciou uma predominância das doenças sistêmicas dentre as doenças reumatológicas. Ao correlacionar o diagnóstico com a idade média das doenças mais prevalentes coletadas (Tabela 2), foi demonstrado uma média de e 52 anos, desta forma, de acordo com os estudos de Widdifield et al (2017) e Dias, Santos, Almeida, Alvares, Guerra, \& Acurcio (2017). Quanto a isso, pode ser observado uma média de idade mais elevada nos pacientes acometidos de osteoartrose, e mais baixa, nas afecções relacionadas a gestação.

A diferença dentre as médias de idade em cada uma das patologias pode ser explicada pelo fato de a osteoartrose ser uma doença crônica de elevada prevalência nos indivíduos idosos, com estimativas de que 5\% dos idosos acima de 70 anos possuam osteoartrose (Corti \& Rigon, 2013). Por outro lado, quando se trata das afecções ligadas a gravidez, a principal investigação dentro da reumatologia é a Síndrome do Anticorpo Antifosfolípide (SAAF), cuja idade de acometimento predominante são as mulheres em idade fértil (Merashli, Noureldine, Uthman, \& Khamashta, 2015) 
Tabela 1 - Pacientes reumatológicos, segundo diagnósticos.

\begin{tabular}{|c|c|c|}
\hline Diagnósticos & $\mathbf{F a}$ & $\operatorname{Fr}(\%)$ \\
\hline Algoneurodistrofia & 1 & 0,55 \\
\hline Artrite idiopática juvenil & 2 & 1,10 \\
\hline Artropatia Psoriásica & 5 & 2,75 \\
\hline Artrite Reumatoide & 37 & 20,33 \\
\hline Artropatia Neuropática & 1 & 0,55 \\
\hline Condromalácia & 1 & 0,55 \\
\hline Dermatopolimiosite Juvenil & 1 & 0,55 \\
\hline Doença Mista do Tecido Conjuntivo & 1 & 0,55 \\
\hline Dor Articular & 1 & 0,55 \\
\hline Esclerose Sistêmica & 4 & 2,20 \\
\hline Espondilite Anquilosante & 1 & 0,55 \\
\hline Febre Reumática & 1 & 0,55 \\
\hline Fibromialgia & 56 & 30,77 \\
\hline Artrite Gotosa & 1 & 0,55 \\
\hline Hepatite Autoimune & 1 & 0,55 \\
\hline Lúpus Discoide & 2 & 1,10 \\
\hline Lúpus Eritematoso Sistêmico & 19 & 10,44 \\
\hline Osteoartrose & 34 & 18,68 \\
\hline Osteoporose & 1 & 0,55 \\
\hline Polimiosite & 1 & 0,55 \\
\hline Síndrome de Sjögren & 1 & 0,55 \\
\hline Afecções Ligadas a Gravidez Não Especificadas & 8 & 4,40 \\
\hline Síndrome do Túnel do Carpo & 1 & 0,55 \\
\hline Sinovite & 1 & 0,55 \\
\hline TOTAL & 182 & 100 \\
\hline
\end{tabular}

Fonte: Acervo da pesquisa (2021).

Tabela 2 - Pacientes Reumatológicos, quanto à idade e sexo.

\begin{tabular}{llll} 
& Idade & Sexo & Masculino \\
\hline Diagnóstico & Média & Feminino & 4 \\
\hline Artrite Reumatoide & 60 anos & 33 & 2 \\
Fibromialgia & 57 anos & 54 & 0 \\
Lúpus Eritematoso Sistêmico & 40 anos & 19 & 2 \\
Osteoartrose & 69 anos & 32 & 0 \\
Afecções Ligadas a Gravidez Não Especificadas & 34 anos & 8 & \\
\hline
\end{tabular}

Fonte: Acervo da pesquisa (2021).

Sobre as comorbidades apresentadas pelos pacientes (Tabela 3), demonstrou-se que, da amostra total de 182 pacientes, 77 pacientes possuem hipertensão arterial sistêmica (HAS), doença de alta prevalência no Brasil compatível com a faixa etária de acometimento das doenças reumatológicas. Em seguida, temos como a segunda comorbidade mais prevalente na amostra coletada, Diabetes Mellitus, também doença de alta prevalência no país. É importante salientar que esta patologia é um fator de risco para processos inflamatórios, pois de acordo com Samuel, Cintra, Sinvieri-Araujo, Sumida, Ferreira, Facundo, 
Azuma e Prieto (2012) os pacientes diabéticos apresentam maiores níveis de concentração plasmática de citocinas próinflamatórias, como a IL-6, IL-17 e TNF- $\alpha$, que podem potencializar quadros de inflamação.

Ainda sobre as comorbidades dos pacientes, é sabido que a grande parte das doenças reumatológicas são crônicas, logo, não possuem cura e sim fator de melhora com o tratamento adequado a cada patologia. Sendo assim, com o uso crônico de medicações e recorrentes idas ao ambiente hospitalar, os pacientes reumatológicos possuem uma maior probabilidade de desenvolver transtornos psiquiátricos em geral. Estudos realizados por Samuel et al (2012), mostram que a incidência de transtorno depressivo maior pode acometer até $47 \%$ dos portadores do lúpus eritematoso sistêmico (LES). Nos dados coletados no centro de especialidade, foram observados que 13 pacientes da amostra total coletadas possuem transtornos depressivos, corroborando com diversos estudos onde é elevada a incidência entre doenças reumatológicas e transtornos psiquiátricos.

Tabela 3 - Pacientes reumatológicos, segundo comorbidades.

\begin{tabular}{|c|c|c|}
\hline Comorbidades & FA & FR $(\%)$ \\
\hline Sem comorbidades & 74 & 40,66 \\
\hline $\begin{array}{l}\text { D. de Alzheimer + Insuficiência Cardíaca Congestiva (ICC) + Hipertensão Arterial Sistêmica } \\
\text { (HAS) + Diabetes Mellitus }\end{array}$ & 1 & 0,55 \\
\hline Asma + HAS + Diabetes Mellitus & 1 & 0,55 \\
\hline Cirrose & 1 & 0,55 \\
\hline D. de Parkinson + Depressão + HAS & 1 & 0,55 \\
\hline Depressão & 6 & 3,30 \\
\hline Depressão + HAS + Diabetes Mellitus & 1 & 0,55 \\
\hline Diabetes Mellitus & 7 & 3,85 \\
\hline Dislipidemia & 2 & 1,10 \\
\hline Dislipidemia + DM & 1 & 0,55 \\
\hline Dislipidemia + DPOC & 1 & 0,55 \\
\hline Dislipidemia + HAS & 2 & 1,10 \\
\hline HAS & 41 & 22,53 \\
\hline HAS + Asma & 1 & 0,55 \\
\hline HAS + Depressão & 5 & 2,75 \\
\hline HAS + Diabetes Mellitus & 12 & 6,59 \\
\hline HAS + Diabetes Mellitus + Dislipidemia + ICC & 1 & 0,55 \\
\hline HAS + Tireoidopatias & 5 & 2,75 \\
\hline HAS + Tireoidopatias + Diabetes Mellitus & 3 & 1,65 \\
\hline Insuficiência Cardíaca Congestiva (ICC) & 1 & 0,55 \\
\hline Neoplasia & 1 & 0,55 \\
\hline Osteoporose & 5 & 2,75 \\
\hline Osteoporose + Dislipidemia + Diabetes Mellitus & 1 & 0,55 \\
\hline Osteoporose + HAS & 3 & 1,65 \\
\hline Osteoporose + Tireoidopatias & 1 & 0,55 \\
\hline Sequelas neurológicas & 1 & 0,55 \\
\hline Tireoidopatias & 3 & 1,65 \\
\hline Total & 182 & 100 \\
\hline
\end{tabular}


Quando à abordagem sobre tratamento adotado (Figura 2), as condutas realizadas pela equipe multidisciplinar do centro de especialidade são compatíveis com a literatura. A doença mais prevalente detectada em nossa amostra é a fibromialgia, não por acaso, o medicamento mais utilizado é a pregabalina - análogo de GABA (ácido gama-aminobutírico), anticonvulsivo e antiepilético também utilizado no tratamento da dor periférica e ansiedade. Para o tratamento agudo da dor associada à fibromialgia, a pregabalina se mostrou bastante eficaz. Apesar do tratamento mais prevalente ser uma monoterapia, rotineiramente os pacientes reumatológicos costumam realizar terapia polimedicamentosa, devido a prevalência de comorbidades associadas a doenças crônicas e, em especial, as doenças reumáticas (Comissão de Artrite Reumatoide, 2011).

Em nossa amostra, a terapia polimedicamentosa mais prevalente é ciclobenzaprina e fluoxetina. A ciclobenzaprina é um relaxante muscular de ação central e é indicado para o tratamento agudo de espasmos musculares com melhora da dor, sensibilidade e limitação da movimentação. Tal medicamento está relacionado dos sinais e sintomas da osteoartrose e artrite reumatoide, respectivamente segunda e terceira patologia reumatológica mais prevalente em nossa amostra. Já a fluoxetina, é um antidepressivo inibidor seletivo de recaptação de serotonina, utilizado para tratamento de depressão - comorbidade comum quando falamos de doença crônica reumática (Graminha, Pinto, Oliveira, \& Carvalho, 2020).

Figura 2 - Pacientes Reumatológicos, segundo tratamento adotado.

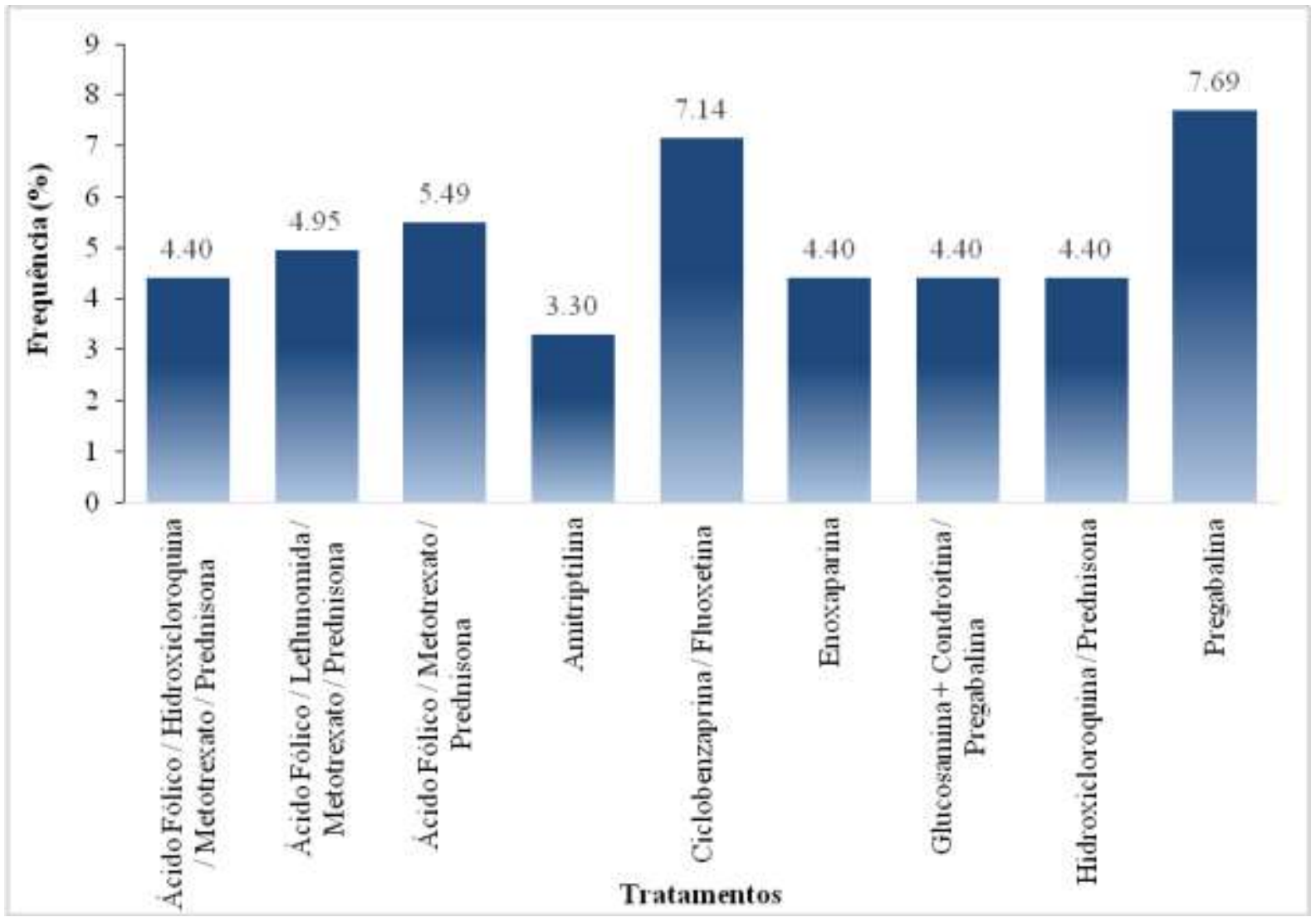

Fonte: Acervo da pesquisa (2021).

Quanto ao tempo de tratamento (Figura 3), observou-se que 31,87\% tinham entre 1 e 2 anos de acompanhamento no serviço, não havendo, na amostra estudada, pacientes com mais de 5 anos de tratamento no centro de estudo. Tais resultados apresentaram-se em contramão aos estudos de Vaz, Faria, Lazarski, Carmo e Sobrinho (2013), o qual demonstrou uma prevalência maior de pacientes com 5 a 9 anos de doença. A discordância com a literatura pode ser explicada devido à implantação recente do sistema de prontuários eletrônicos (e-SUS) ao Centro de Especialidades, o que reduziu o tempo de 
acompanhamento dos pacientes em questão.

Figura 3 - Pacientes Reumatológicos, segundo tempo de tratamento.

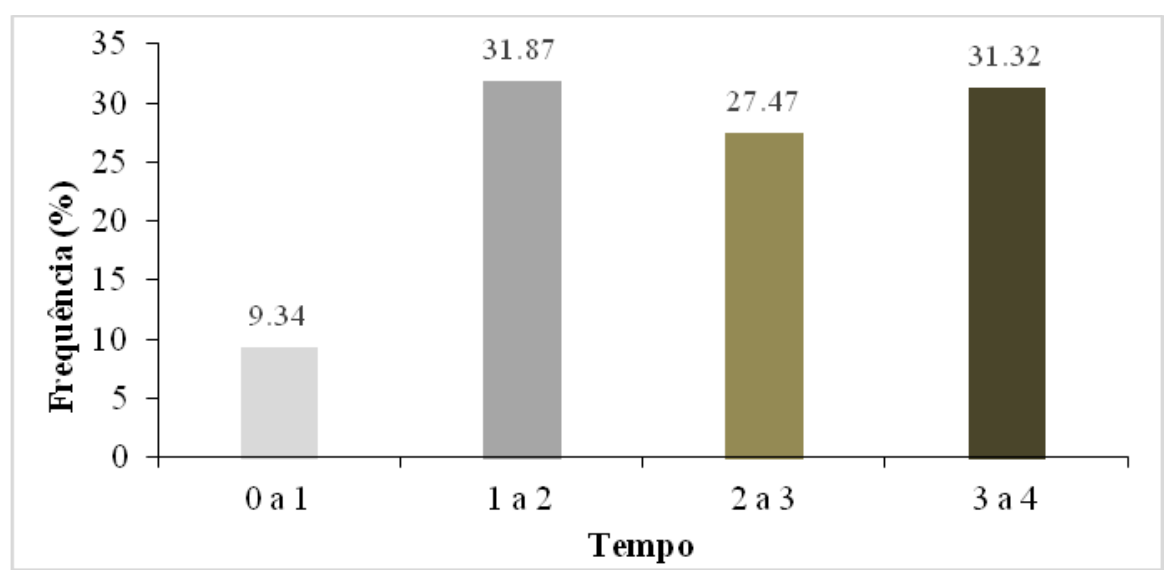

Fonte: Acervo da pesquisa (2021).

\section{Considerações Finais}

Tendo em vista os resultados expostos, fica evidente que o perfil epidemiológico dos pacientes com doença reumática do centro de especialidades localizado no município de Marabá-PA é caracterizado por uma mulher acima de 60 anos, portadora de fibromialgia em uso de gabapentinoides associado ou não a outros medicamentos com menos de 5 anos de tratamento. Com base nisso, podemos observar que o presente estudo está de acordo com os dados epidemiológicos nacionais abordados na literatura, mesmo com uma discordância no tempo de tratamento com os estudos vigentes, o qual pode ser interpretado pela recente implantação do sistema de prontuários eletrônicos (e-SUS) no Centro de Especialidades em questão.

Assim, esta pesquisa possibilita um melhor entendimento dos pacientes acometidos pelas doenças reumatológicas, possibilitando uma melhor abordagem das equipes de saúde para tal população. Além disso, o presente estudo pode auxiliar os órgãos públicos a direcionar os esforços para atender suas demandas, contribuindo para a melhoria da abordagem a esses indivíduos. Uma forma de agregar para futuras pesquisas acerca deste tema seria a adoção de variáveis de etnia, escolaridade e renda, o que torna a caracterização epidemiológica mais completa, abrangendo novos horizontes a serem discutidos para o cuidado com indivíduos portadores de doenças reumatológicas.

\section{Referências}

Bonetti, D. F., Souza, L., \& Resmini, M. B. (2020). Avaliação do perfil epidemiológico de pacientes atendidos no ambulatório de reumatologia das clínicas integradas de uma universidade. Saúde, 46(1), 1-12.

Carvalho, F. M., Costa, M. C. \& Silva, T. C. D. (2013). Doenças reumáticas no Brasil: revisão de estudos epidemiológicos. Efdeportes. Com, Revista Digital, 184(18), 1-1.

Comissão de Artrite Reumatóide. (2011). Comorbidades (Doenças associadas) em pacientes com artrite reumatoide. https://www.reumatologia.org.br/orientacoes-ao-paciente/comorbidades-doencas-associadas-em-pacientes-com-artrite-reumatoide/

Corti, M.C. \& Rigon, C. (2003). Epidemiology of osteoarthritis: Prevalence, risk factors and functional impact. Aging Clin Exp Res 15, 359-363. https://doi.org/10.1007/BF03327356

Costa, R. H. A. (2019). Perfil epidemiológico dos pacientes com Síndrome do Anticorpo Antifosfolípide. Monografia - Programa de Residência Médica do Hospital Geral de Fortaleza, Fortaleza.

Dias, C. Z., Santos, J. B. R., Almeida, A. M., Alvares. J., Guerra, A. A. Jr. \& Acurcio, F. A. (2017). Perfil dos usuários com doenças reumáticas e fatores associados à qualidade de vida no sistema único de saúde, Brasil. Revista Médica de Minas Gerais, 1(27), 1-7.

Estrela, C. (2018). Metodologia Científica: Ciência, Ensino, Pesquisa. Editora Artes Médicas. 
Graminha, C. V., Pinto, J. M., Oliveira, P. A. M. \& Carvalho, E. V. (2020). Relações entre sintomas depressivos, dor e impacto da fibromialgia na qualidade de vida em mulheres. Revista Família, Ciclos de Vida e Saúde no Contexto Social, 8(2), 267-273.

Jorge, M. S. G., Garbin, K., Müller, P. L. \& Wibelinger, L. M. (2017). Atuação fisioterapêutica em um indivíduo com lúpus eritematoso sistêmico associado à artrite reumatoide e à fibromialgia. Abcs Health Sciences, 42(1), 60-64.

Martins, M. de A., Carrilho, F. J., Alves, V. A. F., Castilho, E. A. de, \& Cerri, G. G. (2016). Clínica Médica: doenças endócrinas e metabólicas, doenças ósseas, doenças reumatológicas. Manole.

Melo, G. M. (2018). Alterações nos aspectos biopsicossociais de mulheres com dor crônica causada por artrite reumatoide: Avaliação do relato de dor, qualidade de vida e termografia. TCC (Graduação) - Curso de Terapia Ocupacional, Universidade de Brasília - Faculdade de Ceilândia, Brasília.

Merashli, M., Noureldine, M. H. A., Uthman, I. \& Khamashta, M. (2015). Antiphospholipid syndrome: an update. European Journal Clin Invest. 45 (6), 653662.

Montecucco, C., Cavagna L. \& Caporali R. (2009). Pain and rheumatology: An overview of the problem. European Journal Pain Supplement; 3(2), 105-109.

Oliveira, J. O. Jr. \& Ramos, J. V. C. (2019). Adherence to fibromyalgia treatment: challenges and impact on the quality of life. Brazilian Journal Of Pain, 2(1),81-87.

Rodrigues, A. P., Rodrigues, W. P., Nogueira, T. B. S., Souza, W. J. \& Sousa, M. N. A. (2019). Qualidade de vida em pacientes portadores de doenças reumáticas. Revista Brasileira de Educação e Saúde, 9(1), 06-13.

Rodrigues, R. E., Duarte, P. H. M. \& Feitosa, C. Â. L. (2019). Impacto da osteoartrose de joelho na capacidade funcional e qualidade de vida de pacientes atendidos em um município de Pernambuco, Brasil. Archives Of Health Investigation, 8(7), 361-367.

Samuel, R. O., Cintra, L. TT. A., Sivieri-Araujo, G., Sumida, D. H., Ferreira L. I., Facundo, A. C. S., Azuma, M. M., \& Prieto, A. K. C. (2012), Interrelação entre diabetes, citocinas pró-inflamatórias (IL-6, IL-17 e TNF-alfa) e a presença de periodontites apicais. Rev Odontol UNESP, 41 (esp), 112.

Santos, E. P. Jr., Marson, P. G. \& Nepomuceno, V. R. (2020). Estudo epidemiológico da fibromialgia em ambulatório municipal de reumatologia no Estado do Tocantins. Revista Cereus. 12(3), 259-271.

Silva, A. M. B., Laranjeira, B. M. P., Silva, L. V. C. \& Lorena, S. B. (2017). Avaliação da qualidade de vida de pacientes com doenças reumatológicas. TCC (Graduação) - Curso de Fisioterapia, Centro Universitário Tabosa de Almeida, Caruaru.

Silva, D. R. (2019). Aspectos clínicos, diagnóstico, terapêutico e qualidade de vida de pacientes com artrite reumatoide. Tese (Doutorado) - Curso de Enfermagem, Universidade Federal de Campina Grande-Ufcg, Cajazeiras.

Tien, Y. C., Chiu, Y. M. \& Liu M. P. (2016). Frequency of Lost to Follow-Up and Associated Factors for Patients with Rheumatic Diseases. PLOS ONE, 11(3) 1-10.

Vaz, A. E., Faria, W. A., Lazarski, C. F., Carmo, H. F. \& Sobrinho, H. M. R. (2013). Perfil epidemiológico e clínico de pacientes portadores de artrite reumatóide em um hospital escola de medicina em Goiânia, Goiás, Brasil. Medicina (Ribeirão Preto), 2(46), $141-153$.

Widdifield, J., Tu, K., Carter Thorne, J., Bombardier, C., Michael Paterson, J., Liisa Jaakkimainen, R., Wing, L., Butt, D. A., Ivers, N., Hofstetter, C., Lyddiatt, A., Ahluwalia, V., \& Bernatsky, S. (2017). Patterns of Care Among Patients Referred to Rheumatologists in Ontario, Canada. Arthritis care \& research, 69 (1), 104-114. 